hep-th/0501052

\title{
Direct Proof Of Tree-Level Recursion Relation In Yang-Mills Theory
}

\author{
Ruth Britto, Freddy Cachazo, Bo Feng, and Edward Witten \\ School of Natural Sciences, Institute for Advanced Study, Princeton NJ 08540 USA
}

\begin{abstract}
Recently, by using the known structure of one-loop scattering amplitudes for gluons in Yang-Mills theory, a recursion relation for tree-level scattering amplitudes has been deduced. Here, we give a short and direct proof of this recursion relation based on properties of tree-level amplitudes only.
\end{abstract}

January 2005 


\section{Introduction}

Lately, there has been much renewed progress in understanding tree-level and oneloop gluon scattering amplitudes in Yang-Mills theory. Among other things, a new set of recursion relations for computing tree-level amplitudes of gluons has recently been introduced [1]. These relations express any tree-level amplitude of gluons as a sum over terms constructed from the product of two subamplitudes with fewer gluons times a Feynman propagator. The subamplitudes are physical, on-shell amplitudes with shifted momenta. These recursion relations were deduced by using known properties of one-loop amplitudes to make inferences about tree amplitudes.

A straightforward application of these recursion relations gives new and unexpectedly simple forms for many amplitudes. Many of these very compact forms have been obtained very recently [2, 3, 4] using somewhat related methods.

The recursion relations can be schematically written as follows

$$
A_{n}=\sum_{r} A_{r+1}^{h} \frac{1}{P_{r}^{2}} A_{n-r+1}^{-h} .
$$

Here, for any positive integer $s, A_{s}$ denotes the tree-level scattering amplitudes for $s$ cyclically ordered gluons. In writing a recursion relation for $A_{n}$, one "marks" two of the gluons and sums over products of subamplitudes, with $r$ external gluons on one side, $n-r$ external gluons on the other side, and one internal gluon connecting them, and with the two marked or reference gluons being on opposite sides. (The sum in (1.1) is really a sum over decompositions with one marked gluon on each side, not just a sum over $r$.) $P$ is the momentum and $h$ the helicity of the internal gluon. Momenta are shifted so that this gluon as well as the external ones are on-shell.
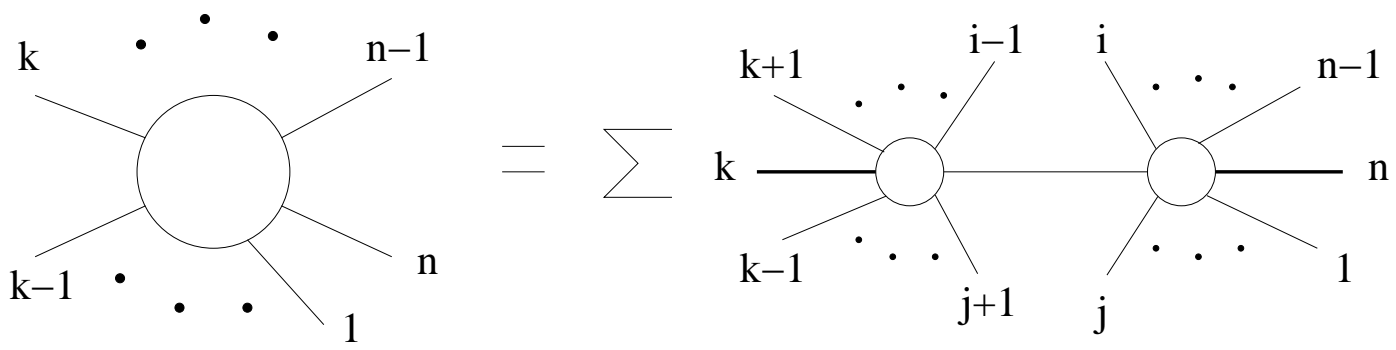

Fig. 1: Pictorial representation of the recursion relation (1.1). Thick lines represent the two marked gluons. The choice of marked gluons here is in anticipation of the discussion in section 2. The sum is over all cyclically ordered distributions of gluons with at least two gluons on each subamplitude and over the two choices of helicity for the internal gluon. 
In [1], an outline of a proof of this formula was given by using a recently discovered method for computing one-loop amplitude in $\mathcal{N}=4$ gauge theory [5] combined with the IR behavior of the amplitudes.

However, the simplicity of the recursion relation (1.1) begs for a more direct and transparent derivation. In particular, one suspects that there should be a derivation that uses properties of tree amplitudes only, rather than deducing properties of tree amplitudes from properties of loop amplitudes.

The aim of this note is to provide such a proof. The proof only uses basic facts about tree diagrams, such as the fact that their singularities come only from poles of internal propagators, plus the description of tree amplitudes via MHV diagrams [6], which we use at one step in the proof to show that the tree amplitudes vanish in a certain limit. (For one arrangement of helicities, we also prove this directly from standard Feynman diagrams.) The recursion relations of [1] use two adjacent gluons of opposite helicity as reference gluons. Our proof shows that the recursion relations can also be defined for reference gluons of the same helicity, and that the gluons do not have to be adjacent.

We conclude this paper by using the BCF recursion relations to show that MHV tree diagrams give the same Yang-Mills tree amplitudes as Feynman diagrams.

\section{Derivation of the BCF Recursion Relations}

Gluon tree amplitudes are most conveniently written using the spinor-helicity formalism [7,8,9]. In a nutshell, the idea is that in four dimensions any null vector $p$ can be written as a bispinor, $p_{a \dot{a}}=\lambda_{a} \widetilde{\lambda}_{a}$. The inner product of vectors can be written in terms of the natural inner product of spinors $\left\langle\lambda, \lambda^{\prime}\right\rangle=\epsilon_{a b} \lambda^{a} \lambda^{\prime b}$ and $\left[\widetilde{\lambda}, \widetilde{\lambda}^{\prime}\right]=\epsilon_{\dot{a} \dot{b}} \tilde{\lambda}^{\dot{a}} \widetilde{\lambda}^{\dot{b}}$. More explicitly, if $q_{a \dot{a}}=\lambda_{a}^{\prime} \widetilde{\lambda}_{\dot{a}}^{\prime}$, then $2 p \cdot q=\left\langle\lambda, \lambda^{\prime}\right\rangle\left[\widetilde{\lambda}, \widetilde{\lambda}^{\prime}\right]$. It turns out that polarization vectors also have a representation in terms of spinors and the full amplitude becomes a rational function of spinor products (for a review see [10]).

Consider a tree-level amplitude $A(1,2, \ldots, n-1, n)$ of $n$ cyclically ordered gluons, with any specified helicities. Denote the momentum of the $i^{t h}$ gluon by $p_{i}$ and the corresponding spinors by $\lambda_{i}$ and $\widetilde{\lambda}_{i}$. Thus, $p_{i}^{a \dot{a}}=\lambda_{i}^{a} \widetilde{\lambda}_{i}^{\dot{a}}$.

In what follows, we single out two of the gluons for special treatment. Using the cyclic symmetry, without any loss of generality, we can take these to be gluons $k$ and $n$. Introduce a complex variable $z$, and let

$$
\begin{aligned}
& p_{k}(z)=\lambda_{k}\left(\widetilde{\lambda}_{k}-z \widetilde{\lambda}_{n}\right) \\
& p_{n}(z)=\left(\lambda_{n}+z \lambda_{k}\right) \widetilde{\lambda}_{n}
\end{aligned}
$$


We leave the momenta of the other gluons unchanged, so $p_{s}(z)=p_{s}$ for $s \neq k, n$. In effect, we have made the transformation

$$
\tilde{\lambda}_{k} \rightarrow \widetilde{\lambda}_{k}-z \widetilde{\lambda}_{n}, \quad \lambda_{n} \rightarrow \lambda_{n}+z \lambda_{k}
$$

with $\lambda_{k}$ and $\widetilde{\lambda}_{n}$ fixed. Note that $p_{k}(z)$ and $p_{n}(z)$ are on-shell for all $z$, and $p_{k}(z)+p_{n}(z)$ is independent of $z$. As a result, we can define the following function of a complex variable $z$,

$$
A(z)=A\left(p_{1}, \ldots, p_{k-1}, p_{k}(z), p_{k+1}, \ldots, p_{n-1}, p_{n}(z)\right) .
$$

The right hand side is a physical, on-shell amplitude for all $z$. Momentum is conserved and all momenta are on-shell.

For any $z \neq 0$, the deformation (2.1) does not make sense for real momenta in Minkowski space, as it does not respect the Minkowski space reality condition $\widetilde{\lambda}= \pm \bar{\lambda}$. However, (2.1) makes perfect sense for complex momenta or (if $z$ is real) for real momenta in signature ++-- . In any case, we think of $A(z)$ as an auxiliary function. In the end, all answers are given in terms of spinor inner products and are valid for any signature. In the derivation of recursion relations, it will be necessary to assume that the helicities $\left(h_{k}, h_{n}\right)$ are $(-,+),(+,+)$, or $(-,-)$. To get a recursion relation in the remaining case $(+,-)$, we use the cyclic symmetry to exchange the roles of $k$ and $n$, or equivalently, we exchange the roles of $\lambda$ and $\tilde{\lambda}$ in (2.1).

$A(z)$ is a rational function of $z$. To see this, note that the original tree-level amplitude is a rational function of spinor products, as we recalled above. Since the $z$ dependence only enters via the shift $\widetilde{\lambda}_{k} \rightarrow \widetilde{\lambda}_{k}-z \widetilde{\lambda}_{n}$ and $\lambda_{n} \rightarrow \lambda_{n}+z \lambda_{k}, A(z)$ is clearly rational in $z$.

In fact, more specifically, for generic external momenta, $A(z)$ has only simple poles as a function of $z$. Singularities come only from the poles of a propagator in a Feynman diagram. As we will see, each propagator gives only a single simple pole, and for generic external momenta, distinct propagators give poles at distinct values of $z$.

To explain these statements, recall first that the momentum flowing through a propagator in a tree diagram is always a sum of external momenta. In Yang-Mills theory, tree diagrams are planar, and the momentum in a propagator is always a sum of momenta of adjacent external particles, say $P_{i j}=p_{i}+\ldots+p_{j}$ for some $i, j$ with $j>i$. A propagator with this momentum is $1 / P_{i j}^{2}$. At nonzero $z$, this becomes $1 / P_{i j}(z)^{2}$ with $P_{i j}(z)=p_{i}(z)+\ldots+p_{j}(z)$. In our problem, as $p_{s}$ is independent of $z$ for $s \neq k, n$, and $p_{k}(z)+p_{n}(z)$ is independent of $z, P_{i j}(z)$ is completely independent of $z$ if both 
$k$ and $n$ or neither of them are in the range from $i$ to $j$. We consider the remaining cases that one of $k, n$ is in this range and the other is not. By momentum conservation, we could replace $p_{i}+\ldots+p_{j}$ by $-\left(p_{j+1}+\ldots+p_{i-1}\right)$. So there is no essential loss of generality in assuming that $n$ is in the range from $i$ to $j$ while $k$ is not. In this case, $P_{i j}(z)=P_{i j}+z \lambda_{k} \widetilde{\lambda}_{n}$, so $P_{i j}(z)^{2}=P_{i j}^{2}-z\left\langle\lambda_{k}\left|P_{i j}\right| \widetilde{\lambda}_{n}\right]$ (where for any spinors $\lambda, \widetilde{\lambda}$ and vector $p$, we define $\left.\langle\lambda|p| \widetilde{\lambda}]=-p_{a \dot{a}} \lambda^{a} \widetilde{\lambda}^{\dot{a}}\right)$. Clearly then, the propagator $1 / P_{i j}(z)^{2}$ has only a single, simple pole, which is located at $z_{i j}=P_{i j}^{2} /\left\langle\lambda_{k}\left|P_{i j}\right| \widetilde{\lambda}_{n}\right]$. For generic external momenta, the $z_{i j}$ for distinct pairs $i, j$ are distinct. These poles are the only singularities of $A(z)$. So $A(z)$, as claimed, has only simple poles.

In section 3 , we use MHV tree diagrams to prove that $A(z)$ vanishes for $z \rightarrow \infty$ as long as the helicities of particles $k$ and $n$ are $(-,+),(+,+)$, or $(-,-)$. (As explained above, in the remaining case, one should make a slight modification of (2.1).) A rational function $A(z)$ that vanishes at infinity and whose only singularities are simple poles at $z=z_{i j}$ has an expansion

$$
A(z)=\sum_{i, j} \frac{c_{i j}}{z-z_{i j}}
$$

where $c_{i j}$ are the residues of the poles. From the above discussion, the sum over $i$ and $j$ runs over all pairs such that $n$ is in the range from $i$ to $j$ while $k$ is not.

The physical scattering amplitude that we want to calculate is simply $A=A(0)$. In terms of the poles and residues, it is

$$
A=-\sum_{i, j} \frac{c_{i j}}{z_{i j}}
$$

This is obtained from (2.4) by setting $z$ to zero in the denominators without changing the numerators. As we will now see, this formula is equivalent to the BCF recursion relation.

In fact, it is easy to describe the residue of the pole at $z=z_{i j}$. To get a pole at $P_{i j}^{2}(z)=0$, a tree diagram must contain a propagator that divides it into a "left," containing all external gluons not in the range from $i$ to $j$, and a "right," containing all external gluons that are in that range. See figure 1. The internal line connecting the two parts of the diagram has momentum $P_{i j}(z)$, and we need to sum over the helicity $h= \pm$ at, say, the left of this line. (The helicity at the other end is opposite.) The contribution of

1 Incidentally, in this argument, there is no need to distinguish collinear singularities (the cases $j=i+1$ and $j=i-3$ ) from multiparticle singularities (the other cases). For complex momenta, these can all be treated alike. 
such a diagram is $\sum_{h} A_{L}^{h}(z) A_{R}^{-h}(z) / P_{i j}(z)^{2}$, where $A_{L}^{h}(z)$ and $A_{R}^{-h}(z)$ are the amplitudes on the left and the right with indicated helicities. Since the denominator $P_{i j}(z)^{2}$ is linear in $z$, to obtain the function $c_{i j} /\left(z-z_{i j}\right)$ that appears in (2.4), we simply must set $z$ equal to $z_{i j}$ in the numerator. When we do this, the internal line becomes on-shell, and the numerator becomes a product $A_{L}^{h}\left(z_{i j}\right) A_{R}^{-h}\left(z_{i j}\right)$ of physical, on-shell scattering amplitudes.

The formula (2.4) for the function $A(z)$ therefore becomes

$$
A(z)=\sum_{i, j} \sum_{h} \frac{A_{L}^{h}\left(z_{i j}\right) A_{R}^{-h}\left(z_{i j}\right)}{P_{i j}(z)^{2}} .
$$

To get the physical scattering amplitude (2.5), we just need to set $z$ to zero in the denominator without touching the numerator. Hence,

$$
A=\sum_{i, j} \sum_{h} \frac{A_{L}^{h}\left(z_{i j}\right) A_{R}^{-h}\left(z_{i j}\right)}{P_{i j}^{2}} .
$$

This is the BCF recursion relation.

\section{Vanishing At Infinity and MHV Diagrams}

In this section, we complete the proof by showing that $A(z)$ vanishes as $z \rightarrow \infty$ if $\left(h_{k}, h_{n}\right)$ is equal to $(-,+),(+,+)$ or $(-,-)$, or more simply if $h_{n}=+$ or $h_{k}=-$.

The proof uses the MHV diagram construction of Yang-Mills tree amplitudes [6]. In this construction, one computes tree amplitudes from tree-level Feynman diagrams in which the vertices are MHV amplitudes, continued off-shell in a suitable fashion, and the propagators are ordinary Feynman propagators. We will present the argument assuming that $h_{n}=+$, in which case, we can make the argument using ordinary ("mostly plus") MHV vertices. For $h_{k}=-$, one makes the same argument using Feynman diagrams with opposite helicity MHV vertices.

As a warmup, let us suppose that the $n$-gluon amplitude of interest is actually an MHV amplitude. Then

$$
A(z)=\frac{\left\langle\lambda_{r}, \lambda_{s}\right\rangle^{4}}{\prod_{i=1}^{n}\left\langle\lambda_{i}, \lambda_{i+1}\right\rangle},
$$

where $\lambda_{n}$ depends on $z$ as in (2.2), while the other $\lambda$ 's are independent of $z$. In (3.1), $r, s$ are the two gluons of negative helicity; the others all have positive helicity. As long as gluon $n$ has + helicity, $\lambda_{n}$ does not appear in the numerator of $A(z)$, which therefore is independent 
of $z$. The denominator, on the other hand, is a non-trivial polynomial in $z$, because of the factors $\left\langle\lambda_{n-1}, \lambda_{n}\right\rangle$ and $\left\langle\lambda_{n}, \lambda_{1}\right\rangle$, at least one of which (depending on $k$ ) has a nontrivial dependence on $z$. Hence, if $h_{n}=+, A_{n}(z) \rightarrow 0$ for $z \rightarrow \infty$. This argument would clearly fail if gluon $n$ had negative helicity. (For an opposite helicity MHV amplitude, a similar argument shows that $A(z)$ vanishes for large $z$ if gluon $k$ has negative helicity.)

Now consider a general MHV tree diagram. Its contribution to the scattering amplitude is a product of off-shell MHV tree amplitudes, times Feynman propagators $1 / P^{2}$. The propagators are independent of $z$ or vanish for $z \rightarrow \infty$; in fact, their behavior was analyzed in section 2. It will suffice, therefore, to show that the product of off-shell MHV tree amplitudes vanishes for $z \rightarrow \infty$. The key point is to show that if we set up the MHV tree diagrams properly, the off-shell continuation used to define the vertices does not spoil the behavior found in the last paragraph.

A lightlike vector $p$ has a factorization $p_{a \dot{a}}=\lambda_{a} \widetilde{\lambda}_{\dot{a}}$, but there is no such factorization for a vector $P$ that is not lightlike. In defining MHV tree diagrams, one needs to define a positive helicity spinor $\lambda$ associated with each internal momentum $P$ in a Feynman graph. To do this one introduces an arbitrary and fixed negative chirality spinor $\eta$ and defines $\lambda_{a}=P_{a \dot{a}} \eta^{\dot{a}}$. The individual MHV tree diagrams give amplitudes that depend on $\eta$, but the sum does not [6].

In our present problem, it is extremely convenient to pick $\eta=\widetilde{\lambda}_{n}$. The point is that this causes $\lambda$ to be independent of $z$ for each internal gluon, a fact that we can show as follows. Each internal momentum $P$ is a sum $p_{i}+p_{i+1}+\ldots+p_{j}$, for some $i$ and $j$. As in section 2, the $z$-dependence, if any, of such a sum is proportional to $\lambda_{k} \widetilde{\lambda}_{n}$, so if $\eta=\widetilde{\lambda}_{n}$, then $\lambda_{a}=P_{a \dot{a}} \eta^{\dot{a}}$ is independent of $z$ for all internal gluons. In this respect, the internal gluons are no different from the external gluons other than gluon $n$. Hence (with our choice of $\eta$ ), in an off-shell MHV amplitude that appears as a vertex in an MHV tree diagram, all $\lambda$ 's except $\lambda_{n}$, whether associated with internal or external gluons, are independent of $z$. With this at hand, the same analysis we used for physical MHV tree amplitudes shows that, with gluon $n$ assumed to have positive helicity, the off-shell MHV vertex containing gluon $n$ vanishes for $z \rightarrow \infty$. The other MHV vertices are clearly independent of $z$. So the product of the MHV vertices vanishes for $z \rightarrow \infty$, and our argument is complete. 


\subsection{Analysis Using Standard Feynman Diagrams}

This argument, though brief, raises the question of whether MHV tree diagrams are essential or the same result can be deduced from more standard methods. Here we will give an alternative argument based on ordinary Feynman diagrams for the case that the helicities are $\left(h_{k}, h_{n}\right)=(-,+)$.

Recall that any Feynman diagram contributing to the amplitude $A(z)$ is linear in the polarization vectors $\epsilon_{a \dot{a}}$ of the external gluons. Polarization vectors of gluons of negative and positive helicity and momentum $p_{a \dot{a}}=\lambda_{a} \widetilde{\lambda}_{\dot{a}}$ can be written respectively as follows,

$$
\epsilon_{a \dot{a}}^{(-)}=\frac{\lambda_{a} \widetilde{\mu}_{\dot{a}}}{[\widetilde{\lambda}, \widetilde{\mu}]}, \quad \epsilon_{a \dot{a}}^{(+)}=\frac{\mu_{a} \widetilde{\lambda}_{\dot{a}}}{\langle\mu, \lambda\rangle}
$$

where $\mu$ and $\widetilde{\mu}$ are fixed reference spinors.

Only the polarization vectors of gluons $k$ and $n$ can depend on $z$. Consider the $k^{t h}$ gluon first. Recall that $\lambda_{k}$ does not depend on $z$ and $\widetilde{\lambda}_{k}(z)$ is linear in $z$. Since $h_{k}=-$, it

follows from $(3.2)$ that $\epsilon_{k}^{(-)}$goes as $1 / z$ as $z \rightarrow \infty$. A similar argument leads to $\epsilon_{n}^{(+)} \sim 1 / z$.

The remaining pieces in a Feynman graphs are the propagators and vertices. It is clear that the vanishing of $A(z)$ as $z \rightarrow \infty$ can only be spoiled by the momenta from the cubic vertices, since the quartic vertices have no momentum factors and the propagators can only vanish for $z \rightarrow \infty$.

Let us now construct the most dangerous class of graphs and show that they vanish precisely as $1 / z$.

The $z$ dependence in a tree diagram "flows" from the $k^{\text {th }}$ gluon to the $n^{\text {th }}$ gluon in a unique path of propagators. Each such propagator contributes a factor of $1 / z$. If there are $r$ such propagators, the number of cubic vertices through which the $z$-dependent momentum flows is at most $r+1$. (If all vertices are cubic, then starting from the $k^{t h}$ gluon, we find a cubic vertex and then a propagator, and so on. The final cubic vertex is then joined to the $n^{\text {th }}$ gluon.) So the vertices and propagators give a factor that grows for large $z$ at most linearly in $z$.

As the product of polarization vectors vanishes as $1 / z^{2}$, it follows that for this helicity configuration, $A(z)$ vanishes as $1 / z$ for $z \rightarrow \infty$. 


\section{Proof Of MHV Recursion Relations}

For the $(+,-)$ helicity pair, we have shown that the generalized amplitude $A(z)$ vanishes at infinity, and hence the BCF recursion relations are obeyed, using either standard Feynman diagrams or the MHV tree diagrams.

It follows that the BCF relations for $(+,-)$ helicity are satisfied for either the amplitudes computed using Feynman diagrams or the amplitudes computed using MHV tree diagrams. The $(+,-)$ relation is enough to determine the amplitude recursively, given that the all + and all - amplitudes vanish. Hence, the MHV tree diagrams give the same amplitudes as the Feynman diagrams.

In making this argument for the MHV tree diagrams, one needs to know that MHV tree diagrams have only the physical singularities of the Feynman diagrams. That the physical singularities appear correctly was shown in section 4 of [6]. Individual MHV tree diagrams have additional unphysical singularities of the form $1 /\left\langle\lambda_{i}, \lambda_{P}\right\rangle$ coming from the MHV vertices. Here $\lambda_{i}$ is the positive chirality spinor of the $i^{t h}$ external particle and $\lambda_{P a}=P_{a \dot{a}} \eta^{\dot{a}}$ is the spinor associated with an off-shell internal particle. These unphysical singularities are located at non-Lorentz invariant points in momentum space (namely $\lambda_{i}^{a} P_{a \dot{a}} \eta^{\dot{a}}=0$ ), so they cancel by virtue of the proof of Lorentz invariance of the sum of MHV tree diagrams given in section 5 of [6]. Indeed, diagrams containing unphysical singularities appear in pairs, and they cancel by the pairwise cancellation that was used in [6] to prove eqn. (5.17) of that paper as part of the proof of Lorentz invariance.

What does the comparison to the BCF recursion relations really add to this discussion? As we have already noted, it was shown in [6] that the MHV tree diagrams generate the same singularities as the Feynman diagrams. (Cancellation of unphysical singularities, though it follows from Lorentz invariance, was not stated explicitly.) Tree amplitudes are completely determined by the singularities they possess when analytically continued to complex momenta. This statement can be proved by the following reasoning. Tree amplitudes are rational functions of the spinor variables $\lambda$ and $\widetilde{\lambda}$. A rational function of complex variables that has no singularities is a polynomial. Hence, letting $A$ denote a tree amplitude computed from Feynman diagrams and $\widetilde{A}$ the corresponding amplitude from MHV tree diagrams, if $\widetilde{A}$ has the correct singularities then $A-\widetilde{A}$ is a polynomial. But on dimensional analysis, no such polynomial is possible for Yang-Mills tree amplitudes with $n>4$ gluons (the dimension of an $n$ gluon tree amplitude is $4-n$ ). For $n=4$ gluons, the validity of the MHV tree diagrams can be checked directly. 
So the equality $A=\widetilde{A}$ essentially follows from the analysis of singularities and Lorentz invariance in [6]. This approach has one drawback: while a tree amplitude is determined by its singularities, there has been until now in Yang-Mills theory no standard, convenient way to actually use the singularities to determine the amplitude. What the BCF recursion relations give us is an extremely convenient way to determine a Yang-Mills tree amplitude from its singularities, making far more satisfying the proof of validity of the MHV recipe based on knowledge of the singularities.

\section{Acknowledgments}

Work of R. Britto, B. Feng and E. Witten was supported by NSF grant PHY-0070928; and that of F. Cachazo was supported in part by the Martin A. and Helen Chooljian Membership at the Institute for Advanced Study and by DOE grant DE-FG02-90ER40542. Opinions and conclusions expressed here are those of the authors and do not necessarily reflect the views of funding agencies. 


\section{References}

[1] R. Britto, F. Cachazo and B. Feng, "New Recursion Relations for Tree Amplitudes of Gluons," hep-th/0412308.

[2] Z. Bern, V. Del Duca, L. J. Dixon and D. A. Kosower, "All Non-Maximally-HelicityViolating One-Loop Seven-Gluon Amplitudes In N = 4 Super-Yang-Mills Theory," hep-th/0410224.

[3] Z. Bern, L. J. Dixon and D. A. Kosower, "All Next-to-Maximally Helicity-Violating One-Loop Gluon Amplitudes in N = 4 Super-Yang-Mills Theory," hep-th/0412210.

[4] R. Roiban, M. Spradlin and A. Volovich, "Dissolving N=4 Loop Amplitudes Into QCD Tree Amplitudes," hep-th/0412265.

[5] R. Britto, F. Cachazo and B. Feng, "Generalized Unitarity And One-Loop Amplitudes In $\mathrm{N}=4$ Super-Yang-Mills," hep-th/0412103.

[6] F. Cachazo, P. Svrček and E. Witten, "MHV Vertices and Tree Amplitudes in Gauge Theory," hep-th/0403047.

[7] F. A. Berends, R. Kleiss, P. De Causmaecker, R. Gastmans and T. T. Wu, "Single Bremsstrahlung Processes In Gauge Theories," Phys. Lett. B103 (1981) 124; P. De Causmaeker, R. Gastmans, W. Troost and T. T. Wu, "Multiple Bremsstrahlung In Gauge Theories At High-Energies. 1. General Formalism For Quantum Electrodynamics," Nucl. Phys. B206 (1982) 53; R. Kleiss and W. J. Stirling, "Spinor Techniques For Calculating P Anti-P $\rightarrow$ W+- / Z0 + Jets," Nucl. Phys. B262 (1985) 235; R. Gastmans and T. T. Wu, The Ubiquitous Photon: Heliclity Method For QED And QCD Clarendon Press, 1990.

[8] Z. Xu, D.-H. Zhang and L. Chang, "Helicity Amplitudes For Multiple Bremsstrahlung In Massless Nonabelian Theories," Nucl. Phys. B291 (1987) 392.

[9] J. F. Gunion and Z. Kunszt, "Improved Analytic Techniques For Tree Graph Calculations And The G G Q Anti-Q Lepton Anti-Lepton Subprocess," Phys. Lett. 161B (1985) 333.

[10] L. J. Dixon, "Calculating Scattering Amplitudes Efficiently," hep-ph/9601359. 